\title{
Predicting Recurrence after a First Unprovoked Venous Thromboembolism: Retrospective Validation of the DAMOVES Score ${ }^{+}$
}

\author{
A. I. Franco Moreno 1,*, M. J. García Navarro 1, C. L. De Ancos Aracil ${ }^{2}$, A. Gimeno García ${ }^{1}$, \\ C. Montero Hernández ${ }^{1}$, A. Villa Martínez ${ }^{1}$, V. Piedrafita Mateo ${ }^{1}$, J. Ortiz Sánchez ${ }^{1}$, \\ I. C. Sanz Acevedo ${ }^{1}$, R. Martín Díaz ${ }^{1}$ and J. M. Ruiz Giardín ${ }^{2}$ \\ 1 Internal Medicine Department, Torrejón University Hospital, Mateo Inurria, s/n (Soto del Henares), \\ 28850 Madrid, Spain; mgarcia@torrejonsalud.com (M.J.G.N.); agimeno@torrejonsalud.com (A.G.G.); \\ cmontero@torrejonsalud.com (C.M.H.); avilla@torrejonsalud.com (A.V.M.); \\ vpiedrafita@torrejonsalud.com (V.P.M.); jortiz@torrejonsalud.com (J.O.S.); \\ isanz@torrejonsalud.com (I.C.S.A.); rmartin@torrejonsalud.com (R.M.D.) \\ 2 Internal Medicine Department, Fuenlabrada University Hospital, Camino del Molino, 2, 28942 Madrid, \\ Spain; cristina.ancos@salud.madrid.org (C.L.D.A.A.); chruizg@gmail.com (J.M.R.G.) \\ * Correspondence: aifranco@torrejonsalud.com; Tel.: +34-916-262-600 (ext. 7210); Fax: +34-914-886-624 \\ + Presented at the 25th Biennial International Congress on Thrombosis, Venice, Italy, 23-26 May 2018. \\ Published: 30 July 2018
}

\begin{abstract}
Unprovoked venous thromboembolism is associated with a 5 to $27 \%$ annual risk of recurrence after discontinuation of anticoagulation, and indefinite anticoagulation is recommended if the bleeding risk is low to moderate. However, in one-third of patients with unprovoked venous thromboembolism, the risk of recurrence is so low ( $<5 \%$ per year) that anticoagulant therapy $>3-6$ months may not be necessary. Several prediction rules were derived to identify patients with unprovoked venous thromboembolism who have a low recurrence risk. In 2016, we presented our results of the original DAMOVES, a nomogram for prediction of recurrence in an individual patient with unprovoked venous thromboembolism. The aim of this study was to externally validate this nomogram in patients with unprovoked venous thromboembolism.
\end{abstract}

Keywords: decision support technique; recurrence; venous thromboembolism

\section{Introduction}

Venous thromboembolism (VTE) is a chronic disease, associated with a significant rate of recurrence which is fatal in 3.6 to $10 \%$ of patients [1]. Anticoagulation (AC) is very effective as recurrent VTE is rarely encountered during this time. However, the price for effective secondary prevention is bleeding. Current guidelines recommend indefinite anticoagulant treatment for all patients with unprovoked proximal deep vein thrombosis and/or pulmonary embolism provided the risk of bleeding is low or moderate and good coagulation monitoring is achievable [2]. It is therefore important to develop strategies to stratify patients according to their recurrence risk, for discrimination between patients in need of long-term secondary prevention from those in whom a short course of anticoagulation is sufficient. Some of the abovementioned risk factors and putative predictors of thrombosis recurrence have been evaluated in order to be incorporated into clinical decision guides aiming at stratifying patients according to their individual high or low risk of recurrence. Several clinical prediction guides have been proposed to this end, drawn from analyses of what happened in prospective cohorts of patients with previous unprovoked VTE [3-5]. In European Journal of Internal Medicine, we published our results of the new simple risk model that 
improves prediction of the recurrence risk in patients with unprovoked VTE [6]. Based on 7 parameters: age, sex, body mass index, D-dimer level during AC, factor VIII coagulant activity, genetic thrombophilia and varicose veins, we developed a nomogram for prediction of recurrence in an individual patient with unprovoked VTE (Figure 1). In our clinical prediction guide, a DAMOVES (D-dimer, $\underline{\text { Age, }}$ Mutation, Obesity, aricose veins, Eight, $\underline{\text { Sex}}$ )] score $<11.5$ points (risk of recurrence $<5 \%$ ), might be sufficient to justify stopping AC in an average patient after 3-6 months of AC. On the other hand, a DAMOVES score $\geq 11.5$ appears to confer a risk of recurrent VTE that warrants prolonged AC, unless a significant bleeding risk is present. We aimed to validate this nomogram in patients with unprovoked VTE.

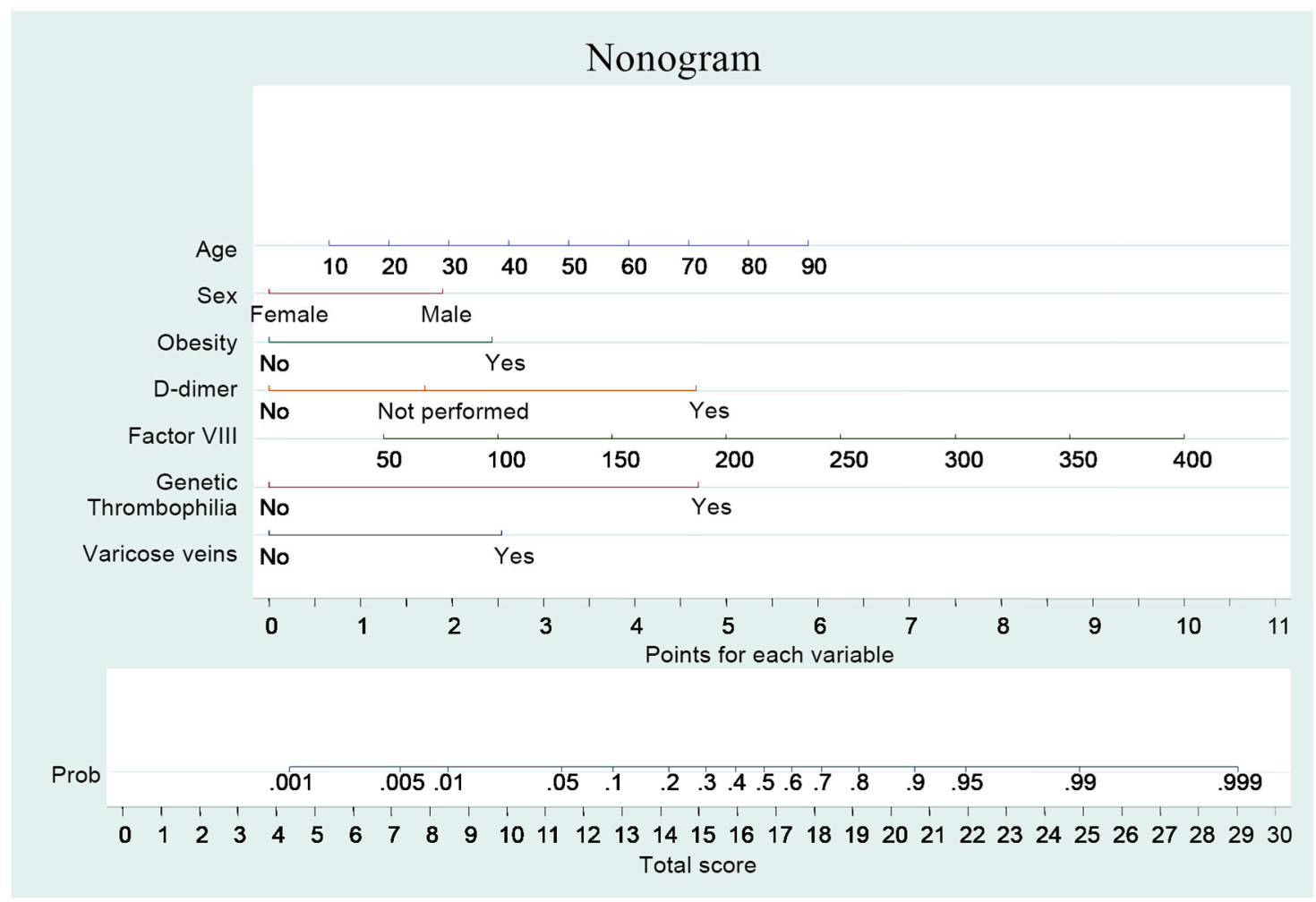

Figure 1. DAMOVES score.

\section{Materials and Methods}

A retrospective review was performed on a cohort of patients with unprovoked symptomatic VTE from department of Internal Medicine of Torrejón University Hospital between August 2012 and October 2015. For the sake of this analysis, only patients with unprovoked VTE and who had completed at least 3 months of AC (heparin followed by acenocoumarol) were included. We determined the proportion of patients classified as low- vs. higher-risk of VTE recurrence according to DAMOVES score. The Receiver Operating Characteristic (AUC) reflects the ability of the model to discriminate between patients with low- vs. higher-risk of VTE recurrence. Stata 14.0 was used for the statistical analyses. In all statistical analyses, a $p$-value $<0.05$ was considered significant.

\section{Results}

A total of 121 patients with unprovoked VTE were included for the analysis. The median followup was 18 months after discontinuation the AC therapy. The proportion of VTE recurrence was $6.61 \%$ (8/121). In all patients, VTE recurred spontaneously. When applying, the cut-off point from the nomogram, 35 (28.92\%) out of 121 subjects were categorized as having a low-risk of recurrent VTE, and $86(71.07 \%)$ as having a high-risk. Table 1 shows the baseline characteristics of development and validation cohorts. Within these two categories, the observed prevalence of recurrent VTE was $2.85 \%$ ( 1 out of 35 subjects) in the low-risk category, and 20\% (7 out of 86 ) in the high-risk category. The 
AUC of the nomogram in the external validation cohort was 0.83 ([95\% confidence interval, $0.743-$ 0.810 ], $p$-value $<0.001)$. The model showed good calibration. The Hosmer-Lemeshow test showed consistent results $(p$-value $=0.125)$.

Table 1. Comparison of baselines characteristics in the development and validation cohorts.

\begin{tabular}{|c|c|c|c|c|c|}
\hline \multirow{3}{*}{ Variables } & \multirow{3}{*}{$\begin{array}{l}\text { Original } \\
(n=398)\end{array}$} & \multicolumn{3}{|c|}{ External Validation } & \multirow{3}{*}{$p$-Value } \\
\hline & & $\begin{array}{c}\text { All } \\
(n=121)\end{array}$ & $\begin{array}{c}\text { Lower-Risk Patients } \\
(n=35)\end{array}$ & $\begin{array}{l}\text { Higher-Risk Patients } \\
(n=86)\end{array}$ & \\
\hline & & \multicolumn{3}{|c|}{$n(\%)$ or Median (Interquartile Range) } & \\
\hline Age (years) & $1.04(1.01 ; 1.07)$ & $72.0(69.0 ; 79.8)$ & $69.0(62.0 ; 77.0)$ & $78.0(69.0 ; 81.0)$ & 0.04 \\
\hline Male sex & $2.89(1.21 ; 6.90)$ & $81.0(66.94)$ & $12.0(34.28)$ & $69.0(80.23)$ & $<0.01$ \\
\hline Obesity (BMI a $\geq 30$ kg/m²) & $3.92(1.75 ; 8.75)$ & $78.0(64.46)$ & $8.0(22.85)$ & $70.0(81.39)$ & $<0.01$ \\
\hline Elevated D-dimer levels during $\mathrm{AC}^{\mathrm{b}}$ & $13.66(4.74 ; 39.37)$ & $69.0(57.02)$ & $5.0(14.28)$ & $64.0(74.41)$ & $<0.01$ \\
\hline Factor VIII coagulant activity & $1.01(1.00 ; 1.02)$ & $128.0(96 ; 181)$ & $135.0(96 ; 151)$ & $171.0(124 ; 181)$ & $<0.01$ \\
\hline Genetic thrombophilia $^{c}$ & $13.86(5.87 ; 32.75)$ & $66.0(54.54)$ & $13.0(37.14)$ & $53.0(61.62)$ & $<0.01$ \\
\hline Varicose veins & $4.14(1.81 ; 9.43)$ & $40.0(33.05)$ & $11.0(31.42)$ & $29.0(33.72)$ & 0.20 \\
\hline
\end{tabular}

a Body mass index; ${ }^{\mathrm{b}}$ Anticoagulation; ${ }^{\mathrm{c}}$ Heterozygous of factor V Leiden and/or Prothrombin G20210A mutation.

\section{Discussion}

We externally validated the DAMOVES score, a clinical prediction guide developed to estimate the risk of disease recurrence in patients with a first unprovoked VTE by using age, sex, body mass index, D-dimer level during AC, factor VIII coagulant activity, genetic thrombophilia and varicose veins as predictors. All variables have been mentioned associated with the recurrence in patients with unprovoked VTE in previously published data. We used a pooled individual patient database from five studies as a validation cohort. The ability of the DAMOVES score to distinguish patients' risk for recurrent VTE in the validation cohort was at least as good as in the original cohort. The robustness of the DAMOVES score in distinguishing between patients at high or low risk of recurrence in the validation cohort is evident, despite differences in patient characteristics in the two cohorts. This study, to our knowledge, is the second external validation of a clinical prediction guide for recurrent VTE risk after the external validation of the Vienna Prediction Model. We developed a prediction score that could be potentially useful to decide whether to continue or stop anticoagulant therapy in patients with a first unprovoked VTE who have received at least 3 months of AC. The score appears to reliably predict recurrence risk and might identify patients with an annual risk of recurrence sufficiently low $(<5 \%$ per year) to justify stopping anticoagulation in patients with unprovoked VTE. There are two potential limitations of our study. First our findings are not applicable to patients with multiple VTE events, with severe thrombophilia, or with VTE occurring in the presence of temporary risk factors (e.g., recent surgery, or estrogen-containing oral contraceptives), or in cancer patients, and second, our model is not applicable to patients using direct oral anticoagulant as anticoagulant therapy.

\section{Conclusions}

In conclusion, our study is an important step towards incorporating a clinical prediction guide for VTE recurrence into routine practice. Further external validation studies and, most importantly, clinical impact studies are now needed to formally assess the impact of the DAMOVES score on clinically relevant outcomes when it is used to decide on the duration of anticoagulation in patients with a first unprovoked VTE.

Author Contributions: A.I.F.M. performed analysis on all samples, interpreted data, wrote manuscript and acted as corresponding author. M.J.G.N., J.O.S. and R.M.D. helped to evaluate and edit the manuscript. C.L.D.A.A., A.G.G., C.M.H., A.V.M., V.P.M. and I.C.S.A. helped in data interpretation. J.M.R.G. supervised development of work and helped to evaluate and edit the manuscript.

Conflicts of Interest: The authors declare no conflict of interest. 


\section{References}

1. Prandoni, P.; Noventa, F.; Ghirarduzzi, A.; Pengo, V.; Bernardi, E.; Pesavento, R.; Iotti, M.; Tormene, D.; Simioni, P.; Pagnan, A. The risk of recurrent venous thromboembolism after discontinuing anticoagulation in patients with acute proximal deep vein thrombosis or pulmonary embolism. A prospective cohort study in 1626 patients. Haematologica 2007, 92, 199-205.

2. Kearon, C.; Akl, E.A.; Ornelas, J.; Blaivas, A.; Jimenez, D.; Bounameaux, H.; Huisman, M.; King, C.S.; Morris, T.A.; Sood, N.; et al. Antithrombotic therapy for VTE disease: CHEST guideline and expert panel report. Chest 2016, 149, 315-352.

3. Rodger, M.A.; Kahn, S.R.; Wells, P.S.; Anderson, D.A.; Chagnon, I.; Le Gal, G.; Solymoss, S.; Crowther, M.; Perrier, A.; White, R.; et al. Identifying unprovoked thromboembolism patients at low risk for recurrence who can discontinue anticoagulant therapy. CMAJ 2008, 179, 417-426.

4. Tosetto, A.; Iorio, A.; Marcucci, M.; Baglin, T.; Cushman, M.; Eichinger, S.; Palareti, G.; Poli, D.; Tait, R.C.; Douketis, J. Predicting disease recurrence in patients with previous unprovoked venous thromboembolism: A proposed prediction score (DASH). J. Thromb. Haemost. 2012, 10, 1019-1025.

5. Eichinger, S.; Heinze, G.; Jandeck, L.M.; Kyrle, P.A. Risk assessment of recurrence in patients with unprovoked deep vein thrombosis or pulmonary embolism: The Vienna prediction model. Circulation 2010, 121, 1630-1636.

6. Franco Moreno, A.I.; García Navarro, M.J.; Ortiz Sánchez, J.; Martín Díaz, R.M.; Madroñal Cerezo, E.; de Ancos Aracil, C.L.; Cabello Clotet, N.; Perales Fraile, I.; Gimeno García, S.; Montero Hernández, C.; et al. A risk score for prediction of recurrence in patients with unprovoked venous thromboembolism (DAMOVES). Eur. J. Intern. Med. 2016, 29, 59-64. 